\title{
Linear Tapered Cavity-Backed Slot Antenna for Millimeter-Wave LTCC Modules
}

\author{
Il Kwon Kim, Student Member, IEEE, Nobutaka Kidera, Stephane Pinel, Member, IEEE, \\ John Papapolymerou, Senior Member, IEEE, Joy Laskar, Fellow, IEEE, Jong-Gwan Yook, Member, IEEE, and \\ Manos M. Tentzeris, Senior Member, IEEE
}

\begin{abstract}
In this letter, a linear tapered slot antenna (LTSA) which is backed by an air cavity is proposed and fabricated using low temperature cofired ceramic (LTCC) substrate. Traditionally, the high dielectric constant and the thick LTCC substrate seriously degrade the gain and radiation property performance because a large portion of the radiated fields are confined in the LTCC substrate. However, the proposed LTSA with the air cavity backing structure exhibits a high gain (4.9-6.9 dBi) and a good end fire radiation pattern. Also, the proposed antenna has a wide impedance bandwidth (about 30 from 45 to $75 \mathrm{GHz}$ ). Through numerical and experimental analyzes, we found that the proposed antenna can be used for a variety of millimeter-wave applications, due to its broadband operating range and its easy integration with 3-D LTCC module.
\end{abstract}

Index Terms-Air cavity backing, endfire pattern, linear tapered slot antenna (LTSA), low temperature cofired ceramic (LTCC), wide bandwidth.

\section{INTRODUCTION}

$\mathbf{N}$ OWADAYS, the interest in antennas operating in the millimeter-wave frequency range has grown significantly, because of numerous broadband emerging applications. The millimeter-wave electronics for commercial applications, such as short-range broadband wireless communications, automotive collision avoidance radars and local cellular radio network (LCRN) require low fabrication cost, excellent performance, and high level integration. To satisfy these needs, low temperature cofired ceramic (LTCC) technology has been extensively studied by many researchers due to its good RF properties, its excellent hermeticity, its mature lamination capability for $12+$ layers and its easy integration with other RFIC technologies, especially for system on package (SOP) modules [1], [2]. However, the high dielectric constant of LTCC material is a challenge for antenna performance [3]. Although the use of high-dielectric constant substrate allows for the realization of compact sized antennas, surface waves and substrate modes

Manuscript received November 22, 2005. revised March 7, 2006. This work was supported in part by KOSEF, the Georgia Electronic Design Center (GEDC), and Asahi Glass Company.

I. K. Kim and J.-G.Yook are with Yonsei University, 120-749 Seoul, Korea (e-mail:kimik@yonsei.ac.kr).

N. Kidera is with the Ashahi Glass Company, 1 Yokohama Kangawa 2218755, Japan (e-mail: nobutaka-kidera@agc.co.jp).

S. Pinel, J. Papaplymerou, J. Laskar, and M. M. Tentzeris are with the School of Electrical and Computer Engineering, Georgia Institute of Technology, Atlanta, GA 77005 USA (e-mail: etentze@ece.gatech.edu).

Digital Object Identifier 10.1109/LAWP.2006.874069

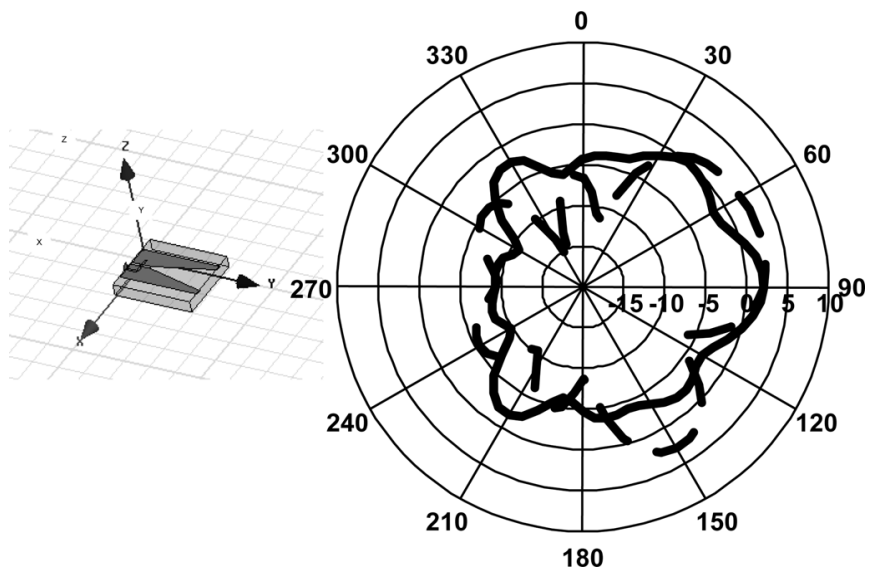

Fig. 1. Radiation pattern of conventional (without cavity back) LTSA on LTCC at $60 \mathrm{GHz}$. [Solid line: azimuth plane (XY). Dashed line: elevation plane $(\mathrm{YZ})]$.

propagating through the dielectric substrate cause edge diffraction effects that further deteriorate the gain and the bandwidth of antenna is significantly degraded.

Linear tapered slot antennas (LTSAs) have been used in systems that require broadband antenna with end-fire radiation patterns. This type of antenna was developed by Gibson et al. [4] and Yngvesson et al. [5], [6]. Although the size of an LTSA is larger than that of a patch antenna in order to generate traveling waves, the impedance bandwidth is wide and endfire patterns are easily achievable. Additionally, this antenna type can be easily fabricated and integrated with other RF circuits on a common board or module. The fabrication of LTSA antennas on LTCC boards is especially challenging, because of the high sensitivity of this antenna to the dielectric thickness as well as the cofiring shrinkage of the LTCC material. The effective thickness range of LTSA to obtain good radiation property is given as $0.005 \lambda o<$ effective thickness $\leq 0.03 \lambda o$ [7]. To alleviate this constraint, several methods have been proposed to lower the effective dielectric constant and thickness of the supporting substrate, such as material modulation [8], [9] or photonic band gap (PBG) [10], [11]. In these methods, air holes are introduced periodically to decrease the permittivity of the dielectric constant. Other approaches consist in etching some portions of the dielectric substrate to achieve a good performance [12], but it is not easy to selectively remove the substrate following the slot shape. In this letter, an LTSA that is backed with an air cavity is proposed to implement an integrated antenna which has a high gain and a broadband impedance matching property. 


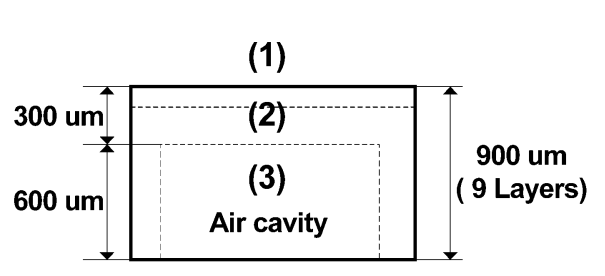

(a) Front View

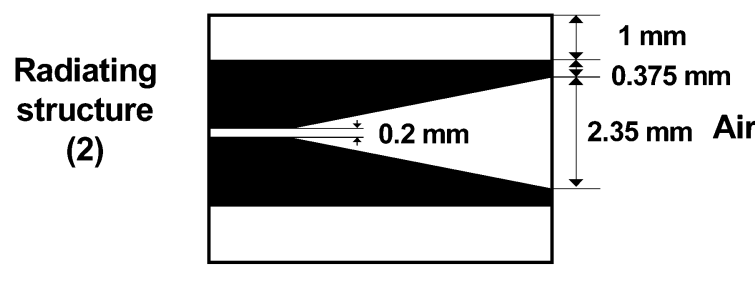

(c) $2^{\text {nd }}$ Layer

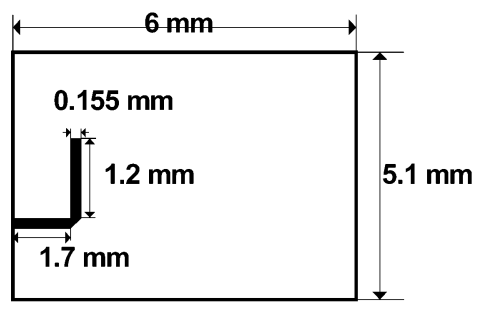

(b) $1^{\text {St }}$ Layer

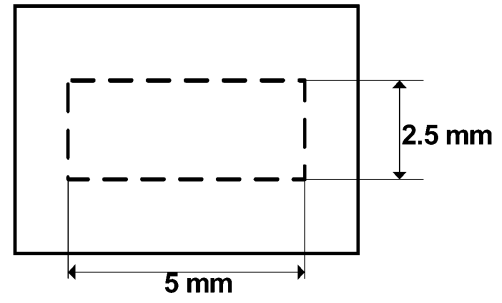

(d) $4^{\text {th }}-9^{\text {th }}$ Layer

Fig. 2. Antenna configuration. (a) Front view (XZ plane). (b) First layer (XY plane). (c) Second layer (XY plane). (d) Fourth-ninth layer (XY plane).

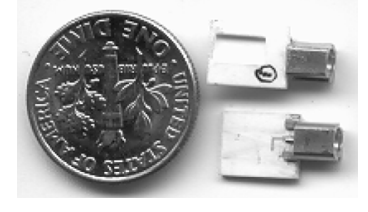

Fig. 3. Fabricated antenna on LTCC substrates.

\section{Linear TAPERED Slot ANTENNA Design On LTCC}

To integrate this antenna to the RF front end module, it is necessary to use a multilayer LTCC structure. This substrate consists of nine total layers that are each $100 \mu \mathrm{m}$ thick. The dielectric constant of the material is 5.4 and its loss tangent is 0.0015.The LTCC substrate we use in this design consists of nine layers and the thickness of each layer (with a permittivity of 5.4) is $100 \mu \mathrm{m}$. Therefore, the total thickness of the substrate is $900 \mu \mathrm{m}$. When one designs an LTSA on LTCC, the radiation pattern is degraded because the field excites substrate modes and surface waves that lead to detrimental diffraction effects. As shown in Fig. 1, the radiation pattern is degraded significantly and the gain of the antenna without the cavity is around $3 \mathrm{dBi}$ at the maximum radiation direction. To alleviate this problem, the proposed antenna consists of three parts: a radiator, a feeding structure, and an air-cavity as shown in Fig. 2. The radiator is a linear tapered slot, which is located in the second layer. The feeding structure is on the first layer to allow for easy measurement and characterization and the radiator is in the second layer to obtain tight coupling with the feeding structure. The feeding structure is a transition from a microstrip-line to a slot-line that is located in the first layer; this structure can be matched easily without an additional matching circuit. The air cavity back is located from the fourth layer to the ninth layer to lower the effective dielectric constant. The size of the air cavity is $5.0 \mathrm{~mm}$ $\times 2.5 \mathrm{~mm}$. The depth of air cavity has been fixed to $600 \mu \mathrm{m}$ to ensure a sufficient mechanical strength and stability. The fabricated antenna which has an air cavity back is shown in Fig. 3 .

Before deciding on the optimum depth of the air cavity, several air cavities $(500 \mu \mathrm{m}, 600 \mu \mathrm{m}, 700 \mu \mathrm{m}$, and $800 \mu \mathrm{m}$ depth) were fabricated on the same LTCC board to investigate the effect of the mechanical strength on the performance of the proposed structure as shown in Fig. 4(a). We define "the deformation" as the difference between the designed depth and the fabricated one. As shown in Fig. 4(b), the deformation is serious for $800 \mu \mathrm{m}$ and $700 \mu \mathrm{m}$ depths of the air cavity. The deformation of $290 \mu \mathrm{m}$ for the $800 \mu \mathrm{m}$ air cavity depth means that there is $290 \mu \mathrm{m}$ additional depth after fabrication (total depth $1090 \mu \mathrm{m}$ ) and $-50 \mu \mathrm{m}$ for 700 means $50 \mu \mathrm{m}$ less depth than the predicted one (total depth $650 \mu \mathrm{m}$ ). As shown in Fig. 4(b), for depth values below $600 \mu \mathrm{m}$, the deformation factor is stable, which means the LTCC process can accurately fabricate the designed cavity depth. Therefore, in this letter, we choose an air cavity depth of $600 \mu \mathrm{m}$ in order to maximize the bandwidth of operation. Although only one example is shown in this letter, we noticed the same deformation on several samples.

\section{CharacterizATION AND Results}

\section{A. Impedance Bandwidth Property of LTSA With Air-Cavity}

The performance of the proposed LTSA antenna has been investigated by use of numerical (HFSS) and experimental methods. Results for the impedance bandwidth are shown in Fig. 5 from 35 to $85 \mathrm{GHz}$. It can be seen that the $\mathrm{S} 11$ bandwidth is about $32 \mathrm{GHz}(43-75 \mathrm{GHz})$ for $10 \mathrm{~dB}$ bandwidth. Although, the impedance bandwidth is wider than $32 \mathrm{GHz}$, the effective bandwidth is approximately $30 \mathrm{GHz}$, because the radiation pattern is distorted at frequencies higher than $75 \mathrm{GHz}$ as explained in the next section.

\section{B. Radiation Property of Proposed LTSA}

By using HFSS, the radiation patterns of the proposed antenna were analyzed for frequencies ranging from 45 to $80 \mathrm{GHz}$ and are shown in Fig. 6. The XY (azimuth) plane and YZ (elevation) plane patterns at $45,50,55,60,65,70,75$, and $80 \mathrm{GHz}$ have been calculated demonstrating that the radiation pattern is distorted at $80 \mathrm{GHz}$. Therefore, we can define the radiation 


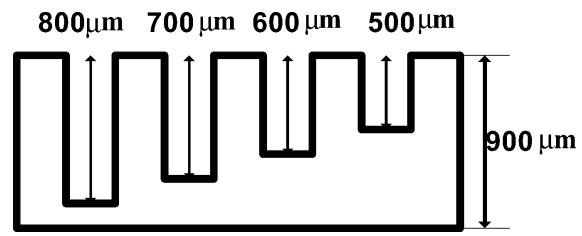

(a)

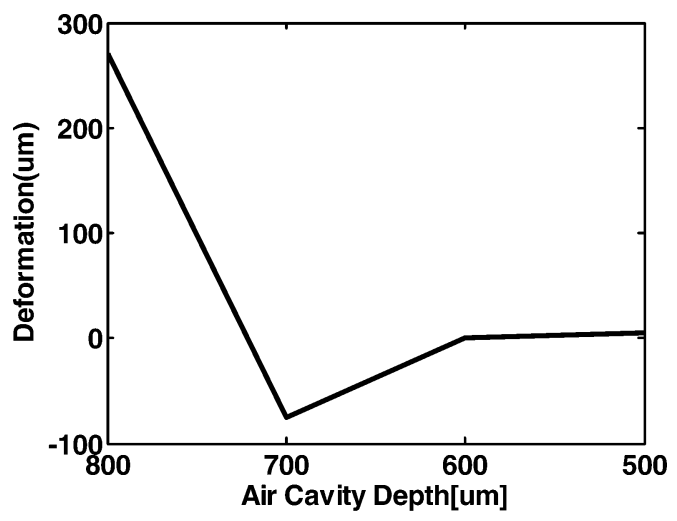

(b)

Fig. 4. Mechanical deformation test for various cavity depths. (a) Cross-sectional view of fabricated cavity. (b) Deformation for various cavity.

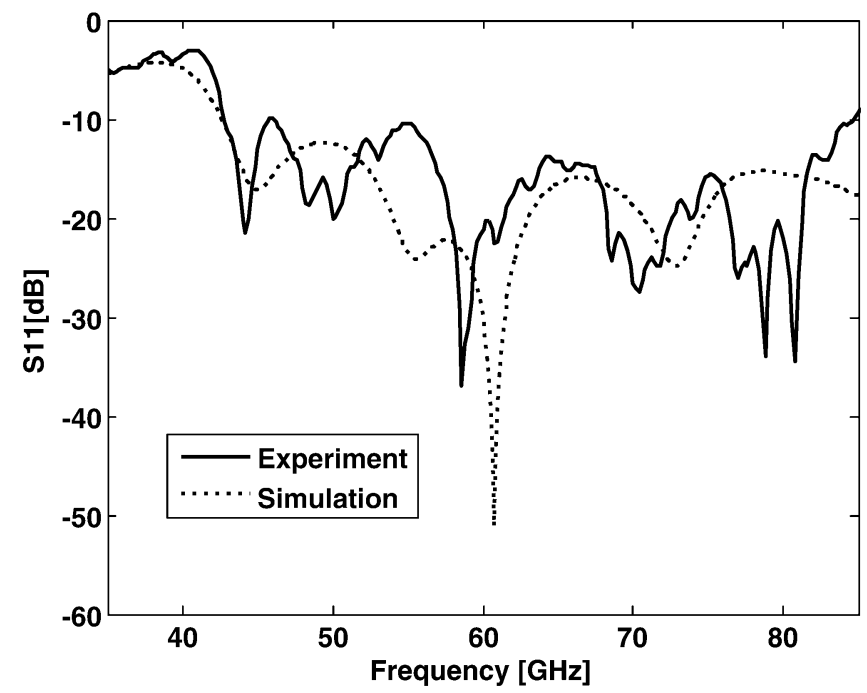

Fig. 5. Simulated and measured results for the return loss of the LTSA.

bandwidth from 45 to $75 \mathrm{GHz}$. It can be seen that the radiation patterns in the elevation plane are asymmetric, while in the azimuth plane, they are symmetric. This is due to the fact that the radiator is on the second layer, so there is one dielectric layer at upper side and two dielectric layers and six air cavity layers at the bottom side. This XY-plane geometrical asymmetry can make the antenna pattern nonsymmetric, especially, from 120 to $180^{\circ}$. Additionally, the gain of this antenna ranges from 5.1 to $6.9 \mathrm{dBi}$ around the center frequency of $60 \mathrm{GHz}$. Comparing with the radiation pattern in Fig. 1, the gain of the cavity backed antenna at $60 \mathrm{GHz}$ is about 3.9-dB higher than that of the antenna without the cavity because the air cavity is adopted to lower the effective dielectric constant and the effective thickness of the LTCC substrate From these results, it is found that the addition
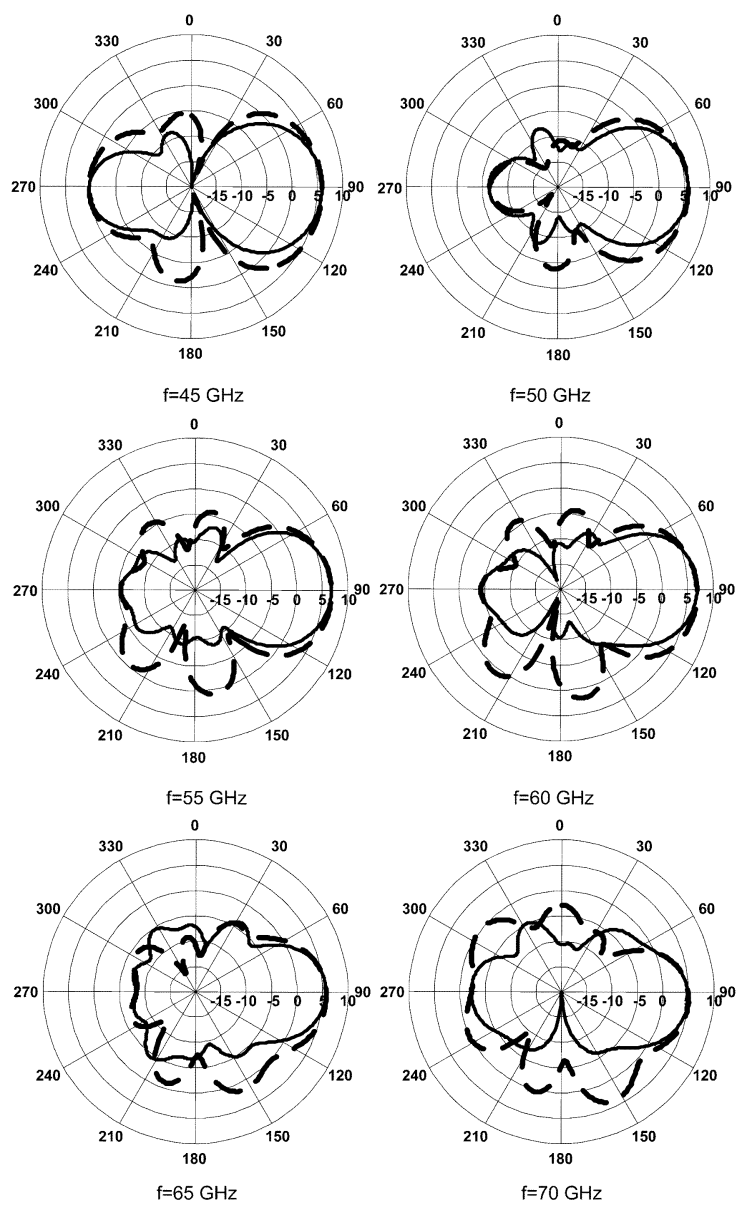

$\mathrm{f}=60 \mathrm{GHz}$

\section{,} . 
TABLE I

GAIN OF PROPOSED ANTENNA

\begin{tabular}{|c|c|c|}
\hline Frequency $[\mathrm{GHz}]$ & Gain $[\mathrm{dBi}]$ & FB $[\mathrm{dB}]$ \\
\hline 45 & 5.9 & 5.6 \\
\hline 55 & 6.8 & 12.1 \\
\hline 65 & 5.5 & 15.2 \\
\hline 75 & 5.8 & 12.2 \\
\hline
\end{tabular}

*FB: Front-Back Ratio [dB]

TABLE II

THE 3 DB BEAMWIDTH [DEG]

\begin{tabular}{|c|c|c|}
\hline $\begin{array}{c}\text { Frequency } \\
{[\mathrm{GHz}]}\end{array}$ & $\begin{array}{c}\text { Elevation plane } \\
\text { (YZ) }\end{array}$ & $\begin{array}{c}\text { Azimuth plane } \\
(\mathrm{XZ})\end{array}$ \\
\hline 45 & 78 & 57 \\
\hline 55 & 57 & 47 \\
\hline 65 & 58 & 47 \\
\hline 75 & 107 & 77 \\
\hline
\end{tabular}

\section{CONCLUSION}

In this letter, an LTSA that is backed with an air cavity is proposed for the high dielectric-constant LTCC technology and its characteristics are investigated up to $80 \mathrm{GHz}$. From simulation and measurement results, it is found that the proposed antenna has a broad bandwidth $(>30 \mathrm{GHz})$ and features a high-gain (above $5 \mathrm{dBi}$ ) end fire radiation pattern. Therefore, this antenna can be used for a variety of millimeter wave applications and can be easily integrated with 3-D LTCC modules.

\section{REFERENCES}

[1] K. Lim, S. Pinel, M. Davis, A. Sutono, C.-H. Lee, D. Heo, A. Obtoynbo, J. Laskar, E. M. Tentzeris, and R. Tummala, "RF system-on-package (SOP) for wireless communications," IEEE Microw. Mag. , pp. 88-99, Mar. 2002.
[2] J.-H. Lee, G. DeJean, S. Sarkar, S. Pinel, K. Lim, J. Papapolymerou, J. Lakar, and M. M. Tentzeris, "High integrated millimeter-wave passive components using 3-D LTCC system-on-Package (SOP) technology," IEEE Trans. Microw. Theory Tech., vol. 53, no. 6, pp. 2220-2229, Jun. 2005.

[3] R. L. Li, G. DeJean, K. Lim, M. M. Tentzeris, and J. Lakar, "Design of compact stacked-patch antennas in LTCC multilayer packaging modules for wireless applications," IEEE Trans. Adv. Packag., vol. 27, no. 4, pp. 581-589, Nov. 2004.

[4] P. J. Gibson, "The vivaldi aerial," in Proc. 9th Eur. Microwave Conf., Brighton, U.K., Jun. 1979, pp. 101-105.

[5] K. S. Yngvesson, "Endfire tapered slot antennas on dielectric substrates," IEEE Trans. Antennas Propagat., vol. 33, no. 12, pp. 1392-1400, Dec. 1985.

[6] K. S. Yngvesson, T. L. Korzeniowski, Y. S. Kim, E. L. Kollberg, and J. F. Johansson, "The tapered slot antenna-A new integrated element for millimeter-wave applications," IEEE Trans. Microwave Theory Tech., vol. 37, no. 2, pp. 365-374, Feb. 1989.

[7] J. B. Muldavin and G. M. Rebeiz, "Millimeter-Wave tapered slot antenna on synthesized low permittvity substrates," IEEE Trans. Antennas Propagat., vol. 47, no. 8, pp. 1276-1280, Aug. 1999.

[8] K. Shuler, Y. Venot, and W. Wiesbeck, "Innovative material modulation for multilayer LTCC antenna design at $76.5 \mathrm{GHz}$ in radar and communication applications," in Proc. 33rd Eur. Microwave Conf., Munich, Germany, Oct. 2003, pp. 707-710.

[9] J. S. Colburn and Y. Rahmat-Samii, "Linear taper slot antenna directivity improvement via substrate perforation: A FDTD evaluation," in IEEE AP-S Dig., Atlanta, GA, Jun. 1998, pp. 1176-1179.

[10] T. J. Ellis and G. M. Rebeiz, "MM-Wave tapered slot antennas on micromachined photonic bandgap dielectrics," in IEEE MTT-S Dig., San Francisco, CA, 1996, pp. 1157-1160.

[11] J. B. Rizk and G. M. Rebeiz, "Millimeter-Wave fermi tapered slot antennas on micromachined silicon substrates," IEEE Trans. Microw. Theory Technol., vol. 50, no. 3, pp. 379-383, Mar. 2002.

[12] U. K. Kotthaus and B. Vowinkel, "Investigation of planar antennas for submillimeter receivers," IEEE Trans. Microwave Theory Technol., vol. 37, no. 2, pp. 375-380, Feb. 1989. 